\title{
Success Of An Expedited Emergency Department Triage Evaluation System For Geriatric Trauma Patients Not Meeting Trauma Activation Criteria
}

This article was published in the following Dove Press journal: Open Access Emergency Medicine

\author{
Forrest B Fernandez (D) \\ Adrian Ong (D) \\ Anthony P Martin' \\ C William Schwab ${ }^{2}$ \\ Tom Wasser (D) ${ }^{3}$ \\ Christopher A Butts ${ }^{4}$ \\ Amanda R McNicholas' \\ Alison L Muller' \\ Charles F Barbera ${ }^{5}$ \\ Rachael Trupp (D) ${ }^{5}$ \\ Adam P Sigal ${ }^{5}$ \\ 'Trauma and Surgical Critical Care \\ Reading Hospital, Reading, PA, USA; \\ ${ }^{2}$ Trauma and Surgical Critical Care, \\ Hospital of the University of \\ Pennsylvania, Philadelphia, PA, USA; \\ ${ }^{3}$ Complete Statistical Services, Macungie, \\ PA, USA; ${ }^{4}$ Philadelphia College of \\ Osteopathic Medicine, Philadelphia, PA, \\ USA; ${ }^{5}$ Department of Emergency \\ Medicine, Reading Hospital, Reading, \\ PA, USA
}

Background: Geriatric patients are at increased risk of injury following low-energy mechanisms and are less tolerant of injury. Current criteria for trauma team activation (TTA) often miss these injuries. We evaluated a novel triage process for an expedited Emergency Medicine Physician evaluation protocol (T3) for at-risk geriatric sub-populations not meeting trauma team activation (TTA) criteria.

Methods: Retrospective review of injured patients ( $\geq 65$ years) from a Level II Trauma Center with an Injury Severity Score (ISS < 16), prior to (Pre-T3, Jan 2007-Oct 2009), and after (Post-T3, Jan 2010-Oct 2012), implementation of T3, as well as a contemporary period (CP, Jan 2013-Oct 2015). Demographics, physiologic variables, and timeliness of care were measured. Rates of ICU admission, operative procedures and lengths of stay and in-hospital mortality were compared for all periods. Logistic regression analysis determined variables independently associated with mortality. Results: Post-T3, 49.2\% of geriatric registry patients underwent T3 with a reduction in key time intervals. Median time to evaluation (42.1 mins vs $61.7 \mathrm{~min}, \mathrm{p}<0.001$ ), median time to CT (161.3 mins vs 212.9 mins, $\mathrm{p}<0.001)$ and EDLOS (364.6 mins vs 451.5 mins, $\mathrm{p}=0.023$ ) were all reduced compared to non-expedited evaluations. There was no change in mortality after the implementation of the protocol.

Conclusion: The T3 protocol expedited patient evaluation of at-risk geriatric patients that would not otherwise meet TTA criteria. The new process met the goals of the American College of Surgeons Trauma Quality Improvement Program while conserving resources.

Keywords: trauma, triage, geriatrics

\section{Introduction}

Older adults, defined as those aged over 65, are at increased risk of significant injury following low-energy traumatic mechanisms and are also less tolerant of physiologic insult. ${ }^{1,2}$ Traditional triage thresholds for trauma activation that rely solely on abnormal vital signs, altered sensorium, anatomic injury, or the presence of a high-energy mechanism often fail to capture significantly injured patients in this population. ${ }^{3,4}$ Prior to November 2009, a significant proportion of older adults presenting to our trauma center did not meet our trauma team activation (TTA) criteria, and were evaluated by an Emergency Medicine physician (EMP), with subsequent consultation to the Trauma Service after injuries were identified. To expedite the evaluation of this vulnerable population, we created a new EMP-led third triage tier. Criteria for patient triage to "Tier 3" (T3) were designed to identify patients with occult head, neck, and torso injury
Correspondence: Adam P Sigal Department of Emergency Medicine, Reading Hospital, 6th Avenue and Spruce Street, P.O. Box 16052, West Reading, PA I96II, USA

Tel +l 4846283636

Email adam.sigal@towerhealth.org 
following low-energy mechanisms without baseline neurologic or physiological derangements meeting TTA criteria.

Our outcomes of interest were time to physician evaluation (TPE), time to computed tomography (TCT), and Emergency Department (ED) length of stay in older patients following lowenergy mechanisms. We hypothesized that the introduction of T3 would have a positive impact on these metrics. Although initiated in 2009, the addition of T3 aligns with the goals of the 2013 American College of Surgeons Trauma Quality Improvement Program (ACS TQIP) Geriatric Trauma Management guidelines. $^{5}$

\section{Methods}

The study took place at a Level II state-accredited trauma center serving a broad urban and rural catchment area of approximately 450,000 people. Blunt mechanisms of injury predominate $(93 \%)$. The center averages 1,500 TTAs, and 2000 trauma service admissions annually. Patients $\geq 65$ years of age make up approximately $40 \%$ of our registry and $15 \%$ of the registry is aged $\geq 85$. Emergency Department (ED) volume exceeds 130,000 contacts annually.

\section{Study Design}

The Reading Hospital Institutional Review Board approved the study. The IRB found that the study was a review and satisfied criteria for Federal regulation 45 CFR $164.512 \mathrm{i}$ with waiver of consent granted. Respect for patient data confidentiality was maintained as all analysis was performed on de-identified patient information. This study was performed in compliance with the Declaration of Helsinki.

We performed a retrospective review from our trauma registry of injured patients aged $\geq 65$ years with an ISS $<16$ meeting Pennsylvania Trauma Outcome Study (PTOS) inclusion criteria (Box 1). ${ }^{6}$ The first period of study (Pre-T3: Jan 2007 - Oct 2009), was prior to the introduction of the T3 protocol, during

Box I Pennsylvania Trauma Outcome Study (PTOS) Inclusion Criteria

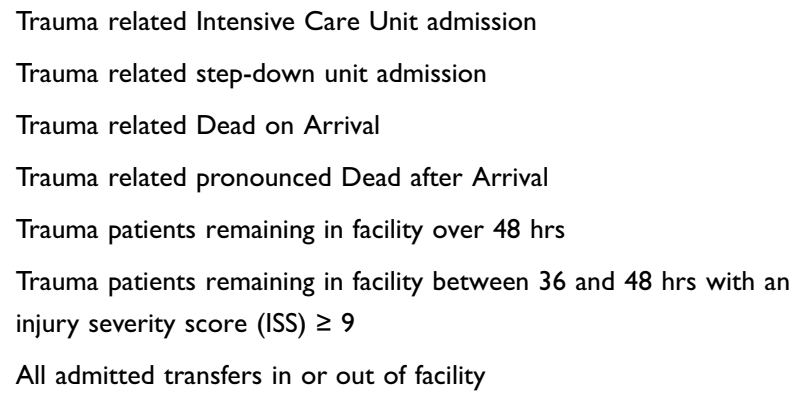

which an EMP evaluated patients not meeting TTA triage criteria. A learning period of 2 months (Nov 2009-Dec 2009) was used to implement the protocol. This study compared PreT3 outcomes with a similar time-frame following completion of the learning period (Post-T3, Jan 2010-Oct 2012), as well as a third comparable contemporary period (CP, Jan 2013-Oct 2015). The contemporary period was included to evaluate the sustainability of the impact on outcomes with T3 implementation.

\section{Triage}

Our center's triage criteria delineate three levels of activation (Figure 1). A "Trauma Alert" (Level 1, highest level) activates the trauma team (Trauma Surgeon, EMP, Resident/Advanced Practitioner, Trauma Nurse (TN) x 2, Paramedic, Laboratory Technician (LT), Radiology Technician (RT), ED Registrar (EDR), and Chaplain) as well as an additional Anesthesia Provider, Respiratory Therapist, and delivery of 2 type-specific units of packed red blood cells (PRBCs). A "Trauma Response" (Level 2) activates the trauma team only without the immediate mobilization of the Anesthesia Provider or blood products. The T3 protocol (Level 3) mobilizes a board-certified EMP, TN, LT, RT, and EDR for expedited evaluation. Patients not meeting entry criteria for the first 3 levels of activation were triaged to a traditional non-expedited EMP evaluation (NE). The T3 evaluation consisted of a standard history and physical with emphasis on the primary and secondary trauma survey. The EMP then decided which advanced imaging studies were needed in addition to standard trauma laboratory studies. The Radiology Department also expedited the acquisition and reading of computed tomography studies ordered under the T3 process. The institution set an a priori goal of $30 \mathrm{mins}$ for TPE, $\leq 90 \mathrm{mins}$ for TCT, and $\leq$ 180 mins for ED LOS.

\section{Descriptive And Outcome Variables}

Table 1 lists descriptive variables from the electronic medical record (EMR). Time interval metrics included time from patient arrival to the initial EMP evaluation (TPE), time to first CT acquisition (TCT), and ED length of stay (EDLOS). Variables were then compared between the three periods. Data elements were missing in $2.8 \%$ of patients and such patients were excluded from the analysis. Upgrades from T3 to TTA were included in the $\mathrm{T} 3$ group, consistent with an intention-totreat principle.

\section{Statistical Analysis}

All statistical analysis was performed using SPSS v24.0 (IBM corp., Armonk NY). Data analysis was conducted using 


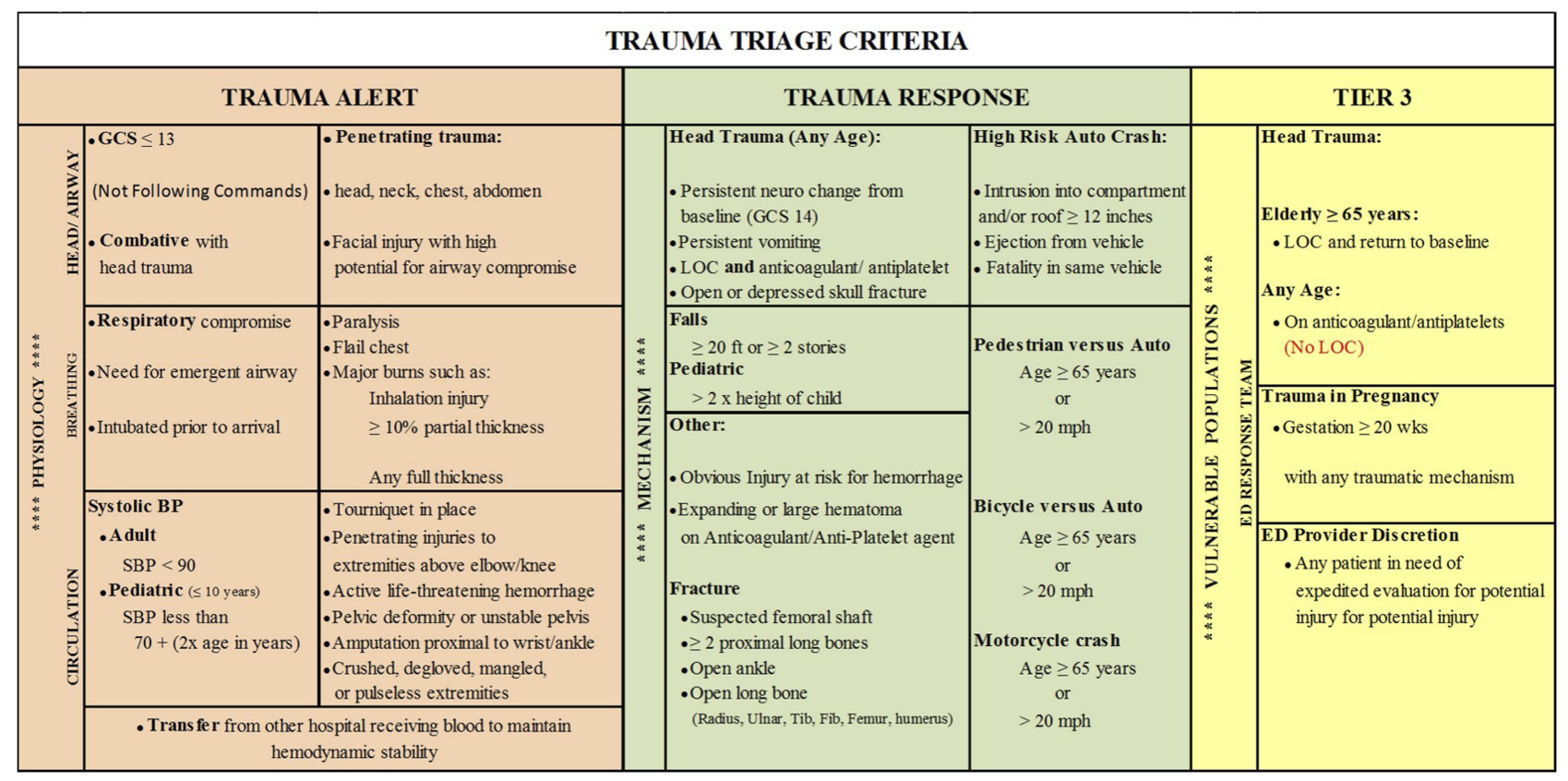

Figure I Three levels of Trauma Activation Criteria. Trauma Alerts and Responses are based on a combination of physiology and mechanism of injury. The addition of Tier 3 includes vulnerable populations.

planned comparisons between periods, Pre-T3, Post-T3 and $\mathrm{CP}$, in order to maintain the sequential integrity of the analysis and control for the experiment-wise error rate. A Chi-square analysis was used for the discreet variables and group $t$-tests for the continuous level variables. Significance was determined by a p-value of less than 0.05 . There were no corrections used for multiple comparisons because some analyses were outcomes related and some analyses were conducted as the goodness of fit measures. Logistic regression was performed using mortality as the dependent variable and assessing the impact of the discrete variables of time-period, anticoagulant use, PRBC transfusion, and male gender (Referents: Pre-T3, no anticoagulated use, no PRBC transfusion and female gender, respectively). Continuous variables were Age, ISS, SBP and HR. The model examined was the enter method which assesses all variables without exclusion, and reports the beta coefficient, p-value, odds ratio and the $95 \%$ confidence interval of each variable. Model diagnostics were the initial model prediction used as a comparator for enhancement, the final model prediction as well as the Nagelkerke $\mathrm{R} 2$ value for all variables and the Hosmer-Lemeshow Goodness of fit statistic.

\section{Results}

Compared to the pre-T3 period, the new triage level resulted in a significant reduction in TPE and overall EDLOS in the post-T3 period (42.1 mins vs $61.7 \mathrm{mins}$ $\mathrm{p}<0.001$ and 364.6 mins. vs 451.5 mins, $\mathrm{p}<0.001$, respectively). All 3-time intervals, TPE, TCT, and EDLOS were significantly reduced in the CP compared to the pre-T3 period $(44.6,125.7$, and 370.0 mins respectively, $\mathrm{p}<0.001$ ) (Table 2).

Overall ED volume increased throughout the three periods of study (Table 1) for our study population. Post-T3, 49.2\% of all admissions with an ISS $<16$ were evaluated via the T3 pathway (CP, 43.8\%). The proportion of patients undergoing TTAs declined after implementation of the protocol. Differences in mean age, admission GCS, admission SBP, and admission HR were not clinically meaningful. There was a change in injury pattern identification with a higher percentage of PTOS patients identified with significant head (AIS Head $\geq 3$ ) and chest (AIS Chest $\geq 2$ ) injuries through the three periods. For ISS $<16$, there was no statistically significant change of in-house mortality with the implementation of the protocol (Pre-T3: 3.7\% vs Post-T3: 2.7\%, and CP: 2.3\%, $\mathrm{p}<0.145)$. There was a statistically significant reduction in overall hospital LOS post-implementation that was sustained in the CP. Utilization of Pan-CT imaging increased significantly after implementation.

Logistic regression analysis incorporating the abovementioned plausible variables showed that advanced age, lower GCS and lower SBP were independent predictors of mortality in this population (Table 3 ). The post T3 period was not associated with a mortality benefit. 
Table I Characteristics And Outcomes In Geriatric Injured PTOS Patients (ISS*<16) Before And After Implementation Of The T3 Protocol

\begin{tabular}{|c|c|c|c|c|c|}
\hline \multirow[t]{3}{*}{ Descriptive Variable } & Pre-T3 & Post-T3 & $\mathbf{C P}$ & \multirow[t]{3}{*}{ p-Value } & \multirow[t]{3}{*}{ ANOVA Post-Hoc } \\
\hline & $\begin{array}{l}|/| / 2007-|0 / 3| / \\
2009\end{array}$ & $\begin{array}{l}1 / 1 / 2010-10 / 31 / \\
2012\end{array}$ & $\begin{array}{l}1 / 1 / 2013-10 / 31 / \\
2015\end{array}$ & & \\
\hline & $N=749$ & $N=\mid 454$ & $N=1632$ & & \\
\hline TTA (\%) & $224(29.9 \%)$ & $365(25.1 \%)$ & $384(23.5 \%)$ & 0.004 & \\
\hline T3 (\%) & $\mathrm{n} / \mathrm{a}$ & $716(49.2 \%)$ & $715(43.8 \%)$ & 0.003 & \\
\hline Mean Age (SD) & $80.66(8.28)$ & $81.16(8.34)$ & $81.81(8.45)$ & 0.017 & Pre vs $C P(p=0.037)$ \\
\hline Mean GCS (SD) & $14.72(1.38)$ & $14.64(1.54)$ & $14.58(1.49)$ & 0.126 & $\mathrm{~N} / \mathrm{A}$ \\
\hline Mean SBP (SD) & $151.48(34.33)$ & 149.83 & $149.49(33.75)$ & 0.416 & $N / A$ \\
\hline Mean Heart Rate (SD) & $80.53(18.46)$ & $80.29(17.91)$ & $79.66(17.56)$ & 0.458 & $N / A$ \\
\hline Male gender (\%) & $284(37.9 \%)$ & $56 \mid(38.6 \%)$ & $632(38.7 \%)$ & 0.929 & \\
\hline Pre-Hospital Anticoagulant Use (\%) & $199(26.6 \%)$ & $4 \mid 4(28.5 \%)$ & $50530.9(\%)$ & 0.071 & \\
\hline ISS Mean (SD) & $7.47(3.70)^{\dagger}$ & $7.60(3.78)^{\ddagger}$ & $7.53(3.60)^{\ddagger}$ & 0.723 & $N / A$ \\
\hline AIS Head $\geq 3$ (\%) & $70(9.3 \%)^{\dagger}$ & $196(13.5 \%)^{\ddagger}$ & $250(15.3 \%)^{\ddagger}$ & $<0.001$ & \\
\hline AIS Chest $\geq 2(\%)$ Region 3 & $206(27.5 \%)^{\dagger}$ & $505(34.7 \%)^{\ddagger}$ & $549(33.6 \%)^{\ddagger}$ & 0.002 & \\
\hline AIS Abdomen $\geq 2(\%)$ Region 4 & $81(10.8 \%)^{\dagger}$ & $168(11.6 \%)^{\ddagger}$ & $173(10.6 \%)^{\ddagger}$ & 0.688 & \\
\hline AIS Ext/Pelvis $\geq 2$ (\%) Region 5 & $365(48.7 \%)^{\dagger}$ & $581(40.0 \%)^{\ddagger}$ & $637(39.0 \%)^{\ddagger}$ & $<0.001$ & \\
\hline \multicolumn{6}{|l|}{ Mechanism of injury } \\
\hline Falls & $613(81.8 \%)$ & $1205(82.9 \%)$ & $1369(83.9 \%)$ & $<0.001$ & \\
\hline Motor Vehicle Crashes** & 91 (12.1\%) & 141 (9.7\%) & $163(10.0 \%)$ & & \\
\hline Pedestrian Struck & $6(0.8 \%)$ & $10(0.7 \%)$ & $22(1.3 \%)$ & & \\
\hline GSW or Stabbing & I (0.1\%) & $6(0.4 \%)$ & $14(0.9 \%)$ & & \\
\hline Other & $38(5.1 \%)$ & $92(6.3 \%)$ & $64(3.9 \%)$ & & \\
\hline ED to ICU (\%) & $288(38.5 \%)$ & $473(32.5 \%)$ & $478(29.3 \%)$ & $<0.001$ & \\
\hline ED to AS (\%) & $7(0.9 \%)$ & $22(1.5 \%)$ & $20(1.2 \%)$ & 0.503 & \\
\hline ED to OR (\%) & $32(4.3 \%)$ & $30(2.1 \%)$ & $54(3.3 \%)$ & 0.011 & \\
\hline ED Transfusion (PRBCs) & $9(1.5 \%)$ & $21(1.4 \%)$ & $26(1.6 \%)$ & 0.759 & \\
\hline Pan CT (\%) & $534(71.3 \%)$ & $120 \mid(82.6 \%)$ & $1319(80.8 \%)$ & $<0.001$ & \\
\hline Intubation & $9(1.2 \%)$ & $15(1.0 \%)$ & $12(0.7 \%)$ & 0.492 & \\
\hline \multicolumn{6}{|l|}{ Outcome Variables } \\
\hline Mortality (\%) & $28(3.7 \%)$ & $39(2.7 \%)$ & $38(2.3 \%)$ & 0.145 & \\
\hline Median Length of Stay (SD) & $5.18(4.48)$ & $4.45(3.35)$ & $4.57(3.52)$ & $<0.001$ & Pre vs Post $(p<0.001)$, Pre vs $C P(p=0.001$ \\
\hline Median Ventilator Days (SD) & $0.18(1.23)$ & $0.10(1.04)$ & $0.13(1.17)$ & 0.371 & \\
\hline
\end{tabular}

Note: ${ }^{*}$ ISS: Injury Severity Score, ${ }^{* *}$ Includes Motorcycle crashes, ${ }^{\dagger}$ Calculated with AIS 90, ${ }^{\ddagger}$ Calculated with AIS 2005.

Abbreviations: PTOS, Pennsylvania Trauma Outcome Study; T3, Tier 3 Protocol; TTA, Trauma Team Activation; GCS, Glasgow Coma Scale; SBP: systolic blood pressure; ISS, Injury Severity Score; AIS, Abbreviated Injury Scale; Ext, extremity; ED, Emergency Department; ICU, intensive care unit; AS, angiography suite; OR, Operating Room; PRBC, packed red blood cells; IQR, interquartile range; Pan-CT, whole-body computed tomography.

\section{Discussion}

The novel triage process met our primary objective of decreasing key metric times in evaluating patients older than age 65 : time to physician evaluation, time to CT scan, and overall emergency department length of stay. Although we did not meet our a priori target goals, the process did result in significant improvements.

The T3 process did not meet our secondary objective of lowering mortality but it did impact overall hospital length of stay when compared to those patients who underwent the standard non-expedited evaluation. This finding is not surprising given the overall lower ISS of the T3 cohort.

In the United States, $13 \%$ of the total population is over 65 and this proportion is expected to increase to over $20 \%$ by $2030 .^{7,8}$ Although the majority of injuries sustained by this subgroup are by low-energy mechanisms, this population currently represents $25 \%$ of all traumarelated admissions $\mathrm{s}^{5,9}$ and a similar proportion of injuryrelated deaths. ${ }^{1,2,10}$ Pre-existing encephalopathy and comorbid conditions often mask clinical presentation, 
Table 2 Timeliness Of Care Before And After Implementation Of T3 Protocol

\begin{tabular}{|l|l|l|l|l|l|l|}
\hline & & Pre-T3 & Post-T3 & CP & p-value* & Post-Hoc $^{\#}$ \\
\hline \multirow{2}{*}{ TPE } & $\mathbf{n}$ & 614 & 1298 & 1443 & & \\
\cline { 2 - 7 } & Mean (SD) & $61.7(87.4)$ & $42.17(67.0)$ & $44.62(57.8)$ & $<0.001$ & Pre vs Post $(p<0.01)$, Pre vs CP $(\mathrm{P}<0.00 I)$ \\
\hline \multirow{2}{*}{ TCT } & $\mathbf{n}$ & 534 & 1201 & 1319 & & \\
\cline { 2 - 7 } & Mean (SD) & $212.9(661.5)$ & $161.3(550.9)$ & $125.7(217.5)$ & 0.001 & Pre vs CP $(\mathrm{P}=0.001)$ \\
\hline \multirow{2}{*}{ EDLOS } & $\mathbf{n}$ & 708 & 1449 & 1632 & & \\
\cline { 2 - 7 } & Mean (SD) & $451.5(376.1)$ & $364.6(277.9)$ & $370.9(303.5)$ & $<0.001$ & Pre vs Post $(\mathrm{P}<0.001)$, Pre vs CP $(\mathrm{P}<0.001)$ \\
\hline
\end{tabular}

Notes: *Omnibus p-value test. ${ }^{*}$ ANOVA analysis performed for three time periods with Scheffe post hoc test.

Table 3 Predictors Of Mortality By Logistic Regression Analysis

\begin{tabular}{|l|l|l|l|l|}
\hline Variable & Coefficient & p-value & Odds Ratio & 95\% Cl \\
\hline Intercept & 1.138 & 0.383 & & \\
Post-T3 Period & 0.605 & 0.057 & 1.831 & $0.982-3.413$ \\
Age & -0.057 & $<0.001$ & 0.945 & $0.916-0.974$ \\
GCS & 0.411 & $<0.001$ & 1.509 & $1.403-1.623$ \\
ISS & -0.051 & 0.106 & 0.95 & $0.993-1.011$ \\
Anticoagulant Use & -0.414 & 0.109 & 0.661 & $0.399-1.096$ \\
PRBC in ED & -0.306 & 0.142 & 0.737 & $0.490-1.108$ \\
SBP & 0.018 & $<0.001$ & 1.018 & $1.011-1.025$ \\
HR & -0.008 & 0.149 & 0.992 & $0.982-1.003$ \\
Male gender & -0.459 & 0.065 & 0.632 & $0.389-1.028$ \\
Fall & 0.057 & 0.963 & 1.059 & $0.552-2.030$ \\
\hline
\end{tabular}

complicating clinical investigation and increasing vulnerability to injury.

Multiple strategies have been proposed for meeting this triage challenge. The ACS TQIP Geriatric Guideline ${ }^{11}$ currently recommends the following: 1) a lowered threshold for transporting injured patients to trauma centers, ${ }^{12-14}$ and 2) Level 1 or 2 conventional activations upon arrival, ${ }^{15} 3$ ) a lower threshold for laboratory $^{7,16,17}$ and radiologic investigation. ${ }^{18,19}$

In our study, we reviewed a novel triage strategy that meets the goals of the ACS TQIP Geriatric Guideline while judiciously using both Emergency Department and trauma resources. The T3 pathway was utilized for evaluation of $43.8 \%$ of all older adult PTOS patients (CP) with ISS $<16$. Despite this high percent, only $21 \%$ of patients triaged to $\mathrm{T} 3$ ultimately had an injury requiring admission to a surgical service (CP). A similar fraction was found to have medical conditions requiring non-surgical inpatient or observational care with the remainder being discharged. This observation suggests that a majority of T3 patients would not benefit from a full TTA as suggested by the ACS-TQIP Guideline.
There was an increase in the number of patients meeting PTOS criteria after implementing the T3 process. The subsequent decrease in the injury severity for extremity/pelvis is most likely a factor of including more patients with lower acuity injuries although meeting PTOS admission criteria.

Multiple studies have been performed evaluating the impact of triage and trauma team activation on geriatric outcomes. $^{15,18-22}$ This study confirms the low sensitivity of triage activation criteria in capturing significant injuries in patients older than 65. It adds to the current understanding of geriatric triage in demonstrating that the goals of the ACS TQIPS can be achieved in a resource responsible manner while improving the quality of care.

Post-T3, the threshold for utilization of computed tomography imaging decreased significantly. Geriatric patients underwent Pan-CT imaging, defined as CT imaging of the brain, cervical spine, chest, abdomen and pelvis, much more frequently in the Post-T3 period. This increase was intentional as the working group initially challenged EMP to maintain a threshold for diagnostic evaluation comparable to the Trauma Service. Though 
highly sensitive for injury identification, the ultimate clinical benefit of aggressive CT imaging has been questioned by several investigators. ${ }^{23-25}$ Post $\mathrm{T} 3$, confirmation of previously occult injuries, such as isolated rib fractures and stable spinal or pelvic fractures may have resulted in an increased number of patients meeting criteria for admission or observation with a lower overall mortality risk. The observed decline in the prevalence of significant extremity injuries, and the increase in significant head and chest injuries post-implementation supports this hypothesis.

\section{Limitations}

Our study has many of the anticipated limitations of a retrospective study. The data analysis is derived from a single-institution registry of only injured patients. The detailed outcomes of non-injured T3 patients are not available. In addition, our results may not be translatable to smaller EDs with a low prevalence of presenting older adult patients. Additionally, the period of study spans almost 10 years, and many improvements to our institution's infrastructure have been implemented over that time. Although a significant mortality decline occurred concurrent with the implementation of the protocol, the observed outcomes may have resulted from other enhancements in care. However, like most improvements in care, such as massive transfusion protocols and damage control surgery apply to the most severely injured, those with ISS $>16$, these changes should not affect our population studied. Further study of long-term mortality and functional outcomes is needed to characterize the ultimate impact of $\mathrm{T} 3$ in improving outcomes.

A potential confounder in this analysis was a conversion from AIS 2005 to AIS 2008 which occurred in January 2012. ISS calculations in the Pre-T3 time period used the former system, while scores Post-T3 used the latter. Some authors have raised the possibility of induced discrepancies by transitioning between versions. ${ }^{26,27}$

\section{Acknowledgments}

The authors express their gratitude to the multidisciplinary providers whose daily dedication has sustained the T3 program. Special thanks go to the RH trauma registrars who daily abstract the data critical to performance improvement of the Trauma Program. In addition, we would like to acknowledge the following individuals for their significant contributions to the study, Kristen Sandel MD, Sudan Butler MSN and Ryan Spinka BS.

\section{Author Contributions}

FBF, AO, CWS, ARM and CFB designed the study along with writing initial manuscript and edits. ARM and RT performed pilot chart review for design phase, had input into original manuscript and edits. APM extracted data for analysis. TW, APM, ALM and APS performed the statistical analysis and composition of Methods section along with input into original manuscript and design of the Tables. FBF, AO, APM, and TW interpreted the results. CFB and APS implemented the program in ED. FBF, AO, CFB, ARM, CAB, APS, CWS, ALM and APM all contributed to critical revisions of the final document. All authors contributed to data analysis, drafting and revising the article, gave final approval of the version to be published, and agree to be accountable for all aspects of the work.

\section{Disclosure}

The authors report no conflicts of interest in this work.

\section{References}

1. Sterling D, O'Connor J, Bonadies J. Geriatric falls: injury severity is high and disproportionate to mechanism. $J$ Trauma. 2001;50:116119. doi:10.1097/00005373-200101000-00021

2. Sattin R, Mullins R. Geriatric Trauma: the continuing epidemic. JAGS. 2002;50:394-395. doi:10.1046/j.1532-5415.2002.50077.x

3. Phillips S, Rond PC III, Kelly SM, Schwartz PD. The failure of triage criteria to identify geriatric patients with trauma: results from the florida trauma triage study. $J$ Trauma. 1996;40(2):278-283. doi:10.1097/00005373-199602000-00018

4. Heffernan DS, Thakkar RK, Monaghan SF, et al. Normal presenting vital signs are unreliable in geriatric blunt trauma victims. $J$ Trauma. 2010;69(4):813-820. doi:10.1097/TA.0b013e3181f41af8

5. Salottolo KM, Mains CW, Offner PJ, Bourg PW, Bar-Or D. A retrospective analysis of geriatric trauma patients; venous lactate is a better predictor of mortality than traditional vital signs. SJTREM. 2013;21:7

6. Pennsylvania Trauma Systems Foundation. Pennsylvania Trauma Systems Foundation Operational Manual for the Data Base Collection System. 12-13. Effective January 2017. Available from: http://ptsf.org/upload/DRAFT_2017_PTOS_Manual_5-1-2017.pdf. Accessed October 15, 2019.

7. United States Department of Health and Human Services. Aging Integrated Database (AGID) State Level Census 2010. Washington, D.C: Administration for Community Living. Available from: http:// www.aoa.gov/aoarot/aging_statistics/index.aspx. Modified May 24, 2016. Accessed February 16, 2017.

8. United States Department of Commerce, Economics and Statistics Administration, U.S. Census Bureau. The Older Population in the United States: 2010-2050. Available from: https://www.census.gov/ prod/2010pubs/p25-1138.pdf. Published May 2010. Accessed February 16, 2017.

9. Matsushima K, Sheaefer EW, Won EJ, Armen SB, Indeck MC, Soybel DI. Positive and negative volume-outcome relationships in the geriatric trauma population. JAMA Surg. 2014;149(4):319-326. doi:10.1001/jamasurg.2013.4834

10. Champion HR, Copes WS, Buyer D, Flanagan ME, Bain L, Sacco WJ. Major trauma in geriatric patients. Am J Public Health. 1989;79 (9):1278-1282. doi:10.2105/ajph.79.9.1278 
11. American College of Surgeons. ACS TQIP geriatric trauma management guidelines. Avaialable from: https:/www.facs.org/ /media/files/ quality\%20programs/trauma/tqip/geriatric\%20guide\%20tqip.ashx. Accessed January 17, 2017.

12. Ichwan B, Darbha S, Shah MN, et al. Geriatric-specific triage criteria are more sensitive than standard adult criteria in identifying need for trauma center care in injured older adults. Ann Emerg Med. 2015;65 (1):92-100. doi:10.1016/j.annemergmed.2014.04.019

13. Calland JF, Ingraham AM, Martin N, et al. Evaluation and management of geriatric trauma: an eastern association for the surgery of trauma practice management guideline. J Trauma Acute Care Surg. 2012;73(suppl 4):S345-S350. doi:10.1097/TA.0b013e318270191f

14. DeLa'O CM, Kashuk J, Rodriguez A, Zipf J, Dumire RD. The geriatric trauma institute: reducing the increasing burden of senior trauma care. Am J Surg. 2014;208:988-994. doi:10.1016/j. amjsurg.2014.08.007

15. Demetriades D, Sava J, Alo K, et al. Old age as a criterion for trauma team activation. J Trauma. 2001;51:754-757. doi:10.1097/00005373200110000-00022

16. Callaway DW, Shapiro NI, Donnino MW, Baker C, Rosen CL. Serum lactate and base deficit as predictors of mortality in normotensive elderly blunt trauma patients. J Trauma. 2009;66(4):1040-1044. doi:10.1097/TA.0b013e3181895e9e

17. Blow O, Magliore L, Claridge JA, Butler K, Young JS. The golden hour and the silver day: detection and correction of occult hypoperfusion within 24 hrs improves outcome from major trauma. J Trauma. 1999;47(5):964. doi:10.1097/00005373-199911000-00028

18. Kohn MA, Hammel JM, Bretz SW, Stangby A. Trauma team activation criteria as predictors of patient disposition from the emergency department. Acad Emerg Med. 2004;11:1-9. doi:10.1111/j.15532712.2004.tb01364.x
19. Rogers A, Rogers F, Bradburn E, et al. Old and undertriaged: a lethal combination. Am Surg. 2012;8:711-715.

20. Demetriates D, Kariaiskakis M, Velmahos G, et al. Effect on outcome of early intensive management of geriatric trauma patients. Br J Surg. 2002;89(Oct):(10);1319-1322.

21. Hammer PM, Storey AC, Berll T, et al. Improving geriatric trauma outcomes: a small step toward a big problem. J Trauma Acute Care Surg. 2016;81(1):162-167. doi:10.1097/TA.0000000000001063

22. Shifflette VK, Lorenzo M, Mangram AJ, Truitt MS, Amos JD, Dunn EL. Should age be a factor to change from a level II to a level I activation? J Trauma Injury, Infect Crit Care. 2010;69(1):88-92. doi:10.1097/TA.0b013e3181e291e9

23. Tillou A, Gupta M, Baraff LJ, et al. Is the use of pan-computed tomography for blunt trauma justified? A prospective evaluation. $J$ Trauma Injury, Infect Crit Care. 2009;67(4):779-787. doi:10.1097/ TA.0b013e3181b5f2eb

24. Gupta M, Schriger DL, Hiatt JR, et al. Selective use of computed tomography compared with routine whole body imaging in patients with blunt trauma. Ann Emerg Med. 2011;58(4):407-416. doi:10.1016/j.annemergmed.2011.06.003

25. Healy DA, Hegarty A, Feeley I, Clarke-Moloney M, Grace PA, Walsh SR. Systematic review and meta-analysis of routine total body CT compared with selective CT in trauma patients. Emerg Med J. 2014;31:101-108. doi:10.1136/emermed-2012-201892

26. Ringdal KG, Hestnes M, Palmer CS. Differences and discrepancies between 2005 and 2008 abbreviated injury scale versions-time to standardize. Scand J Trauma, Resus. 2012;20:11:1-3.

27. Tohira H, Jacobs I, Matsuoka T, Ishikawa K. Impact of the version of abbreviated injury scale on injury severity characterization and quality assessment of trauma care. J Trauma. 2011;71(1):56-62. doi:10.1097/TA.0b013e31821e5a25
Open Access Emergency Medicine

\section{Publish your work in this journal}

The Open Access Emergency Medicine is an international, peerreviewed, open access journal publishing original research, reports, editorials, reviews and commentaries on all aspects of emergency medicine. The manuscript management system is completely online and includes a very quick and fair peer-review system, which is all easy to use. Visit http://www.dovepress.com/testimonials.php to read real quotes from published authors. 